11. Uhm J, Hamad N, Michelis FV, et al. The risk of polyomavirus BK associated hemorrhagic cystitis after allogeneic hematopoietic SCT is associated with myeloablative conditioning, CMV viremia and severe acute GVHD. Bone Marrow Transplant 2014; 49: 1528-34.

12. Jacobi J, Prignitz A, Büttner M, et al. BK viremia and polyomavirus nephropathy in 352 kidney transplants; risk factors and potential role of mTOR inhibition. BMC Nephrol 2013; 14: 207.

DOI: https://doi.org/10.1016/j.pathol.2019.05.007

\section{Development of monomorphic B- cell plasmacytoma post-transplant lymphoproliferative disorder in a patient with previous autologous stem cell transplant}

Sir,

Post-transplant lymphoproliferative disorders (PTLDs) are uncommon lymphoid and/or plasmacytic proliferations that can occur in patients after undergoing solid organ or haematopoietic stem cell transplantation, secondary to extrinsic immunosuppression inherent in these procedures. In the context of haematopoietic stem cell transplantation, the large majority of reported PTLDs occur in patients who received allogenic haematopoietic stem cell transplantation (AlloHSCT). Only a few cases of PTLD following autologous haematopoietic stem cell transplantation (AHSCT) have been reported. ${ }^{1-6}$ We herein report the rare interesting case of an Epstein-Barr virus (EBV) negative monomorphic B-cell plasmacytoma post-transplant lymphoproliferative disorder (PTLD), developing in a patient who received AHSCT for treatment of advanced stage classic Hodgkin lymphoma (CHL).
A 23-year-old male presented to our institution 4 years previously with fever, weight loss and shortness of breath of 2-4 months duration. Positron emission tomography (PET) scan performed showed a fluorodeoxyglucose (FDG) avid, large, bulky, $10.6 \mathrm{~cm}$ anterior mediastinal mass, multiple FDG avid lymph nodes above and below the diaphragm, as well as multiple FDG lesions in the axial skeleton. A biopsy of the anterior mediastinal mass was performed, and the patient was diagnosed with nodular sclerosis CHL (Fig. 1A). The patient was treated with six cycles of adriamycin, bleomycin, vinblastine and dacarbazine (ABVD) and radiotherapy to the mediastinum. The patient achieved complete remission based on positron emission tomography (PET) scan findings after the treatment. However, 2 years later he developed new FDG avid lymphadenopathy above and below the diaphragm, as well as new FDG avid iliac bone lesions. Biopsy of the iliac bone showed disease relapse of CHL. The patient received platinum-based salvage chemotherapy for the disease relapse. After harvesting of peripheral blood stem cells, he underwent AHSCT. The conditioning regimen consisted of carmustine, etoposide, cytarabine and melphalan. Unmanipulated haematopoietic stem cells were administered at a cell dose of $2.78 \times 10^{6} \mathrm{CD} 34^{+}$cells $/ \mathrm{kg}$. The patient had an episode of culture negative neutropenic fever post-AHSCT, from which he subsequently recovered. He was not put on any immunosuppressive drug regimen post-AHSCT. Three and a half months following AHSCT, the follow-up PET scan showed new FDG avid left submental and level II cervical lymph nodes. An excision biopsy of a left level II cervical lymph node was performed. On histology, the cervical lymph node architecture was preserved with presence of reactive lymphoid follicles. There were increased numbers of small mature plasma cells noted in the paracortical areas and sinuses (Fig. 1B). No neoplastic Reed-Sternberg cells were seen. The histological diagnosis

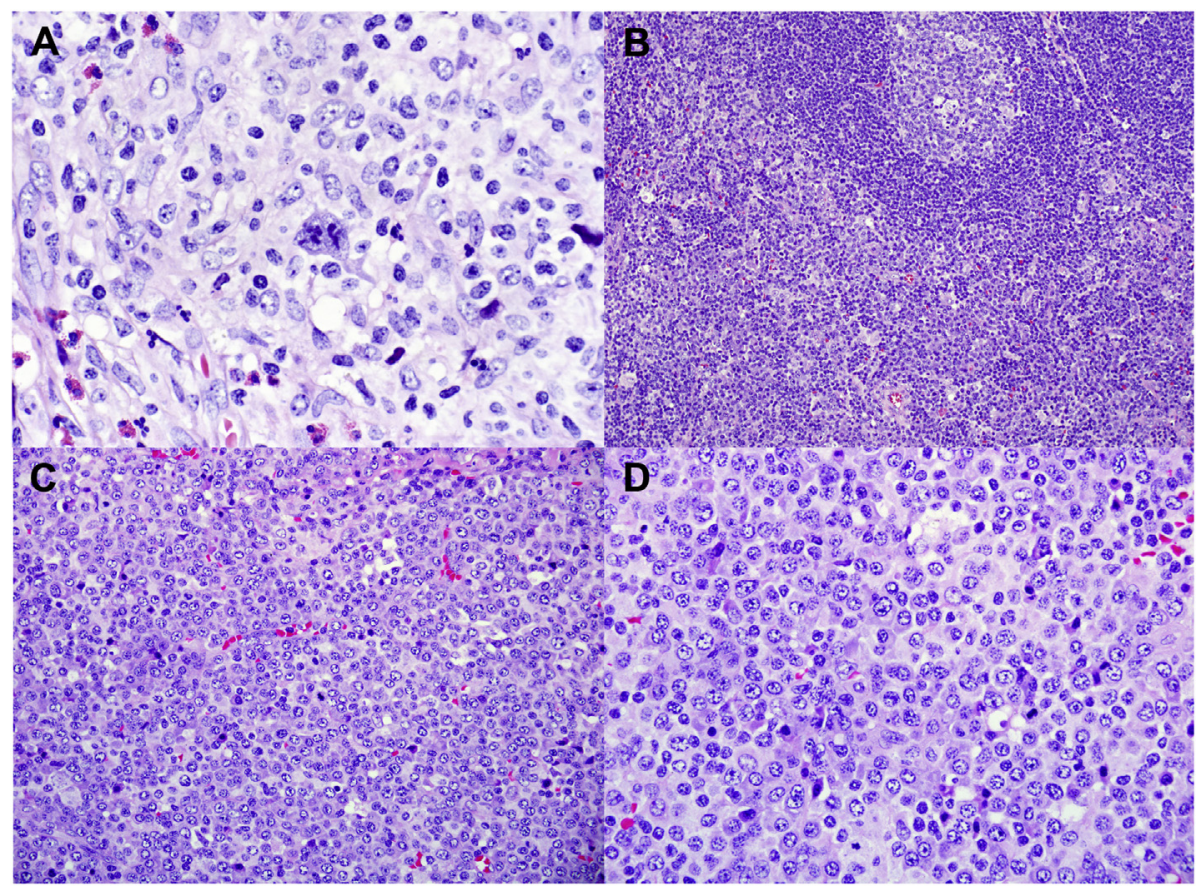

Fig. 1 (A) First mediastinal mass biopsy showing neoplastic classical Reed-Sternberg cells surrounded by reactive inflammatory cells (H\&E). (B) Subsequent left level II cervical lymph node biopsy showing preserved nodal architecture, but with increased numbers of plasma cells in the paracortical areas (H\&E). (C,D) Final left submental lymph node biopsy showing effacement of nodal architecture by sheets of mature plasma cells and plasmablasts (H\&E). 
at that time was a lymph node with reactive changes. However, the patient had a persistently enlarged submental lymph node on follow-up, which was excised 2 months later. On histology, the submental lymph node showed notable architectural effacement by confluent sheets of small mature plasma cells and medium to large lymphoid cells with eccentric nuclei and conspicuous nucleoli, consistent with plasmablasts (Fig. 1C,D). Immunohistochemical (IHC) studies were performed (Fig. 2A-G). The plasma cells and plasmablasts were positive for CD79A and CD138. Only a small proportion of plasmablasts expressed CD20 and CD30. The plasma cells and plasmablasts were negative for CD3, PAX5 and CD56. Kappa and lambda light chains encoded RNA in situ hybridisation was performed. The plasma cells and plasmablasts showed evidence of lambda light chain restriction, indicating a monoclonal mature B-cell population. EBV encoded RNA (EBER) in situ hybridisation was also performed. The plasma cells and plasmablasts showed no evidence of EBV infection. The Ki-67 proliferative index of the plasmacytic/plasmablastic infiltrate was up to $\sim 50 \%$. Immunoglobulin heavy chain $(\operatorname{IgH})$ gene rearrangement study performed using BIOMED2 primers showed a monoclonal gene rearrangement (Fig. 2H). A final diagnosis of an EBV-negative monomorphic B-cell plasmacytoma PTLD was made. Bone marrow studies, bone skeletal survey and other blood investigations performed excluded the diagnosis of myeloma. A subsequent PET scan showed localised disease, comprising mild uptake FDG-avid small volume lymph nodes, confined to the cervical region. The patient was otherwise clinically well with no B symptoms of fever, night sweats and weight loss. After a multi-disciplinary tumour board discussion, the decision was made for watchful waiting with no chemotherapy given for his PTLD. Three months later, follow-up PET scan surprisingly showed resolution of FDG-avid lymph nodes with minimal uptake seen. The resolution was attributed to the patient's immune reconstitution.

PTLDs are lymphoid or plasmacytic proliferations that can occur as a result of immunosuppression after solid organ and haematopoietic stem cell transplant (HSCT). The four main categories of PTLDs are non-destructive (early), polymorphic, monomorphic and classic Hodgkin lymphoma. PTLDs are more often EBV positive, but can also be EBV negative. In contrast to EBV positive PTLDs,

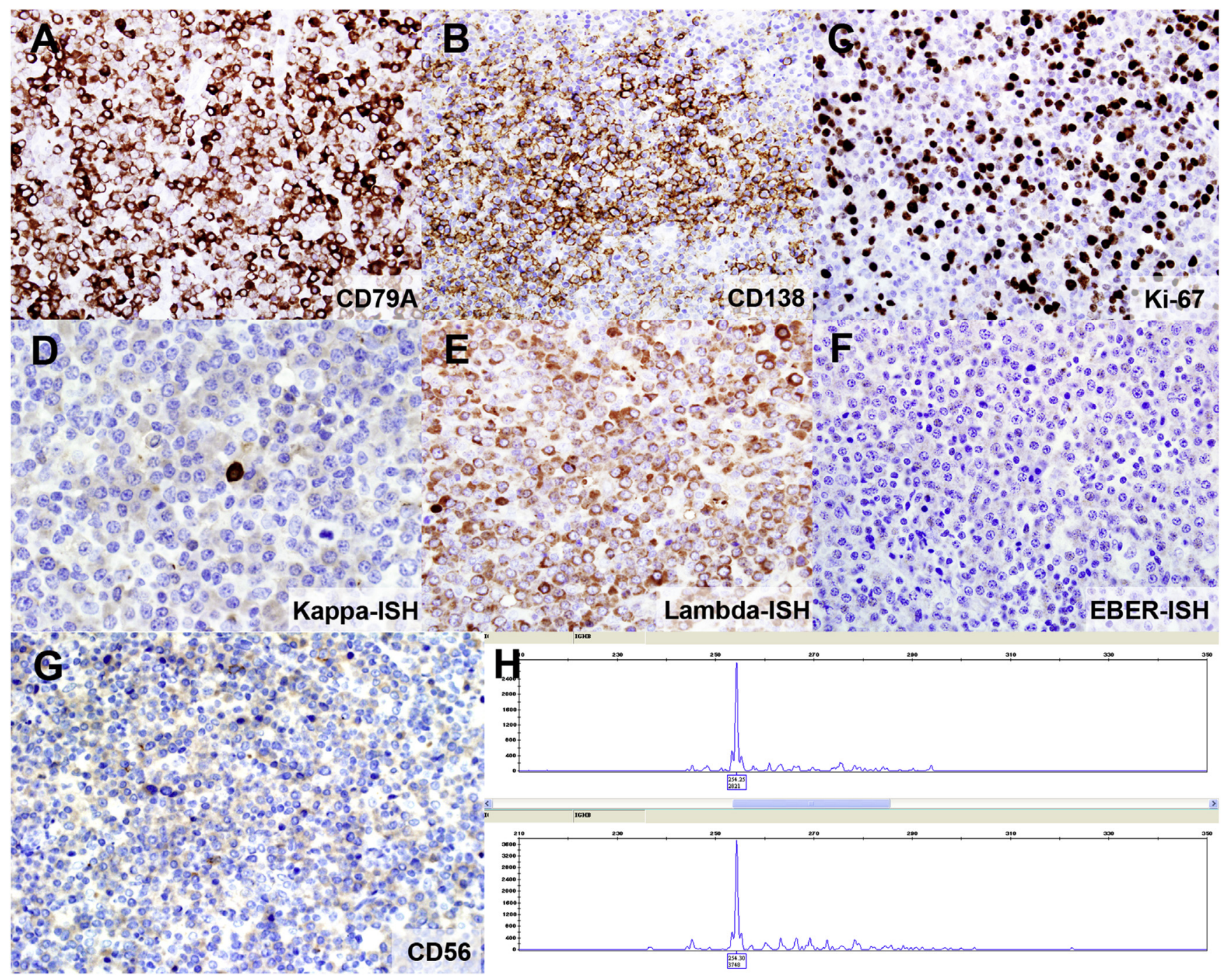

Fig. 2 (A) The plasma cells and plasmablasts are positive for CD79A and (B) CD138. (C) The Ki-67 proliferative index is up to 50\%. (D) Kappa-ISH and (E) lambda-ISH: the plasma cells and plasmablasts show evidence of lambda light chain restriction. (F) The plasma cells and plasmablasts are negative for EBER-ISH and (G) CD56. (H) A monoclonal gene rearrangement is detected on immunoglobulin heavy chain (IgH) gene clonality assay by polymerase chain reaction. 
EBV negative cases are more common in adults, of later onset after transplant, and are more likely to be of monomorphic subtype. ${ }^{8}$ Most cases of PTLD developing after HSCT occurred in the first 6 months post-transplant. There have only been a few reported cases, mostly in the form of case reports, of PTLDs developing after AHSCT as compared to AlloHSCT. This is unsurprising as AHSCT involves a lesser degree of immune suppression than AlloHSCT. The large majority of reported cases of PTLDs developing after AHSCT are EBV positive. Only one case of an EBV negative PTLD with features of Hodgkin lymphoma has been reported. ${ }^{3}$ To the best of our knowledge, ours is the first reported case of an EBV negative monomorphic B-cell plasmacytoma PTLD following AHSCT. The main differential diagnosis considered for our case was a de novo primary nodal plasma cell neoplasm/ plasmacytoma. The diagnosis of PTLD, rather than a de novo plasmacytoma, was supported by disease presentation within 6 months following AHSCT and the apparent improvement of disease without chemotherapy. The disease improvement was attributed to the gradual full restoration of the patient's immune system after the immunosuppressive effects of the AHSCT had dissipated. The histological findings of plasmacytic hyperplasia with preserved nodal architecture in the initial cervical lymph node biopsy specimen were most likely on hindsight a form of nondestructive PTLD. This was followed by progression into a monomorphic PTLD with architectural effacement, as evident in the subsequent submental lymph node biopsy specimen. Non-destructive PTLDs, in contrast with the other subtypes, are defined as lymphoid or plasmacytic proliferations that do not show architectural effacement and do not meet any diagnostic criteria of any known lymphoid neoplasms. The old term of 'early PTLD' was dropped in the revised 4th Edition World Health Organization (WHO) classification to avoid confusion with various PTLDs that occur with an early onset after transplantation. ${ }^{7}$ Nondestructive PTLDs occur more commonly in younger patients with no prior EBV infection. Polymorphic PTLDs show features of architectural effacement, but do not meet diagnostic criteria of any known lymphoid neoplasms. The histological features of monomorphic PTLDs on the other hand will meet the diagnostic criteria of known lymphoid neoplasms as seen in immunocompetent patients. The survival outcomes and treatment modalities of PTLDs are highly variable. The non-destructive PTLDs generally have good clinical response to just reduction in immune suppression. On the other hand, polymorphic and monomorphic PTLDs, besides reduction in immune suppression, may need additional treatment like radiotherapy, surgery, anti-CD20 rituximab drug therapy, immunotherapy and other forms of systemic chemotherapy for disease control. The type of treatment is dependent on the exact PTLD histological subtype, age, disease stage and other blood investigations like lactate dehydrogenase levels.

Plasmacytoma PTLD is a rare form of monomorphic B-cell PTLD that is more commonly reported in adult patients. The histological features are similar to those of plasmacytoma in immunocompetent patients, and are usually composed of sheets of plasma cells with light chain restriction. Some cases also have smaller foci of large lymphoid cells with nucleoli resembling plasmablasts. Many cases of plasmacytoma PTLD have had good clinical outcomes even with limited therapy. ${ }^{10,11}$ The resolution of plasmacytoma PTLD in our case, attributed to the patient's immune reconstitution, supported those findings. Various treatment options of plasamcytoma PTLD include reduction of immune suppression, localised radiation, surgery and systemic chemotherapy based on myeloma protocols. ${ }^{10,11}$

In summary, we present a case of EBV negative monomorphic B-cell plasmacytoma PTLD in a patient who received AHSCT for advanced stage CHL. It is important for pathologists and treating haematologists to diagnose and recognise PTLDs, as their first line of treatment is usually reduction in immune suppression. Some forms of PTLD also have good prognosis even with minimal to limited therapy.

Conflicts of interest and sources of funding: The authors state that there are no conflicts of interest to disclose.

\section{Ming Liang Oon ${ }^{1}$, Gwyneth Soon ${ }^{1}$, Li Mei Poon ${ }^{2}$, Shi Wang}

${ }^{1}$ Department of Pathology, National University Hospital, National University Health System, Singapore; ${ }^{2}$ Department of Haematology-Oncology, National University Hospital, National University Health System, Singapore

Contact Shi Wang.

E-mail: shi_wang@nuhs.edu.sg

1. Awaya N, Adachi A, Mori T, et al. Fulminant Epstein-Barr virus (EBV)-associated T-cell lymphoproliferative disorder with hemophagocytosis following autologous peripheral blood stem cell transplantation for relapsed angioimmunoblastic T-cell lymphoma. Leuk Res 2006; 30: 1059-62

2. Jenkins D, DiFrancesco L, Chaudhry A, et al. Successful treatment of post-transplant lymphoproliferative disorder in autologous blood stem cell transplant recipients. Bone Marrow Transpl 2002; 30: 321-6.

3. Zambelli A, Lilleri D, Baldanti F, et al. Hodgkin's disease as unusual presentation of post-transplant lymphoproliferative disorder after autologous hematopoietic cell transplantation for malignant glioma. BMC Cancer 2005; 5: 109.

4. Lohrisch CA, Nevill TJ, Barnett MJ, et al. Development of a biologically distinct EBV-related lymphoproliferative disorder following autologous bone marrow transplantation for an EBVnegative post-renal allograft Burkitt's lymphoma. Leuk Lymphoma 2000; 39: 195-201.

5. Lones MA, Kirov I, Said JW, et al. Post-transplant lymphoproliferative disorder after autologous peripheral stem cell transplantation in a pediatric patient. Bone Marrow Transplant 2000; 26: 1021-4.

6. Peniket AJ, Perry AR, Williams CD et al. A case of EBV-associated lymphoproliferative disease following high-dose therapy and CD34purified autologous peripheral blood progenitor cell transplantation. Bone Marrow Transplant 1998; 22: 307-9.

7. Swerdlow SH, Campo E, Harris NL, et al. WHO Classification of Tumours of Haematopoietic and Lymphoid Tissues. Revised 4th ed. Lyon IARC Press, 2017; 453-62.

8. Nelson BP, Nalesnik MA, Bahler DW, et al. Epstein-Barr virus negative post-transplant lymphoproliferative disorders: a distinct entity? Am J Surg Pathol 2000; 24: 375-85.

9. Heslop HE. How I treat EBV lymphoproliferation. Blood 2009; 114 4002-8.

10. Trappe R, Zimmermann H, Fink S, et al. Plasmacytoma-like posttransplant lymphoproliferative disorder, a rare subtype of monomorphic B-cell post-transplant lymphoprolifertion, is associated with a favourable outcome in localized as well as in advanced disease: a prospective analysis of 8 cases. Haematologica 2011; 96: 1067-71.

11. Perry AM, Aoun P, Culter DW, et al. Early onset, EBV(-)PTLD in pediatric liver-small bowel transplantation recipients: a spectrum of plasma cell neoplasms with favorable prognosis. Blood 2013; 121 $1377-83$.

DOI: https://doi.org/10.1016/j.pathol.2019.05.008 